\title{
Elementos para una genealogía del movimiento neoliberal en Argentina: intelectuales, políticos y empresarios
}

\author{
Elements for a genealogy of the neoliberal movement in \\ Argentina: intellectuals, politicians and businessmen
}

Elementos para uma genealogia do movimento neoliberal na Argentina: intelectuais, políticos e empresários

\section{María Paula de Büren*}

\begin{abstract}
RESUMEN
Diversos autores entienden que el neoliberalismo encuentra uno de sus momentos organizacionales centrales en la Mont Pèlerin Society. Este trabajo presenta algunos elementos que permiten observar el arribo de esta asociación internacional al territorio argentino. Un proceso que detenta, en el espacio local, la misma configuración y objetivos que asume en el espacio internacional, a saber: vincula grandes empresarios, por un lado, con instituciones y actores que destinan sus esfuerzos a producir y difundir un entramado discursivo y ,por otro, con hacedores de políticas públicas con objeto de influir en el devenir de las naciones o, más precisamente, facilitar la expansión de nuevo ordenamiento social, el cual, la Mont Pèlerín Society ha decidido nominar Neoliberalismo.
\end{abstract}

\section{ABSTRACT}

Several authors understand that neoliberalism finds one of its central organizational moments in the Mont Pèlerin Society. This work presents some elements that allow us to observe this international association's arrival in Argentine territory. It is a process that has the same configuration and objectives in the local space
Palabras clave: empresarios, intelectuales, neoliberalismo, tanques de pensamiento.

Keywords: businessmen, intellectuals, neoliberalism, think tanks.

\footnotetext{
* Doctora en Ciencias Sociales por la Universidad de Buenos Aires. Universidad de Buenos Aires (Facultad de Ciencias Sociales - CBC) y Universidad Nacional de José C. Paz, Buenos Aires, Argentina. E-mail: pauladeburen@yahoo.com.ar
} 
as it has in the global area, namely: it links large businesspeople, on the one hand, with institutions and actors who devote their efforts to producing and disseminating a discursive framework and, on the other, with public policymakers to influence the future of nations or, more precisely, facilitating the expansion of the new social order, which the Mont Pèlerin Society has decided to call neoliberalism.

\section{RESUMO}

Diversos autores entendem que o neoliberalismo encontra um de seus momentos organizacionais centrais na Mont Pèlerin Society. Este trabalho apresenta alguns elementos que nos permitem observar a chegada desta associação internacional ao território argentino. É um processo que apresenta, na esfera local,

Palavras-chave: empresários, intelectuais, neoliberalismo, think tanks. a mesma configuração e objetivos que assume na esfera internacional. Isto é: liga os grandes empresários, por um lado, com instituições e atores que dedicam seus esforços para a produção e disseminação de uma estrutura discursiva e, por outro, com os formuladores de políticas públicas com o objetivo de influenciar o futuro das nações ou, mais precisamente, de facilitar a expansão da nova ordem social, a qual a Mont Pèlerin Society decidiu chamar de Neoliberalismo. 


\section{Introducción}

Numerosos autores entienden que el movimiento neoliberal encuentra dos momentos centrales de articulación: el Coloquio Walter Lippmann (1938) y la gestación de la Mont Pèlerin Society ${ }^{1}$ (1947) (Anderson, 2003; Denord, 2002; Foucault, 2007a; Harvey, 2007, Murillo, 2015b). Estos espacios, desarrollados en un siglo caracterizado por disputas enmarcadas en la Guerra Fría, han reunido a empresarios, intelectuales, políticos y periodistas con objeto de enfrentar en el plano discursivo el avance del comunismo y de todo tipo de intervención estatal de la economía que intente, en alguna medida, recomponer la posición del sector obrero frente al capital, tome aquella la forma de socialismo, keynesianismo, Estado de bienestar, modelos de industrialización, populismo, etc.

Documentos de la sociedad relevan las principales estrategias seguidas por la Mont Pèlerin Society para la consecución de dichos objetivos: la escritura de un nuevo liberalismo, la construcción de una membresía distinguida, la celebración de reuniones periódicas, la creación de una red internacional de personas encargadas de la difusión de ideas liberales y de instituciones liberales dedicadas al seguimiento de las políticas públicas de alta dirección y, finalmente, la intervención política directa (Büren de, 2015a; Hartwell, 1995). La Mont Pèlerin Society necesitaba, para enfrentarse a las formas de organización social adversarias a los intereses de quienes representaba no sólo su deslegitimación a través de la crítica, sino además la escritura y difusión, en todos los rincones del globo, de un nuevo liberalismo con el objeto de influir de manera efectiva en el quehacer político real.

Con miras a la consecución de tales objetivos y a través del accionar de sus integrantes, la Mont Pèlerin Society ingresa en universidades y da inicio a instituciones dedicadas a la difusión discursiva de las interpretaciones del mundo, la historia y la realidad local que considera propicia y afines a sus objetivos. La historia oficial de la Mont Pèlerin Society, A history of the Mont Pèlerin Society $(1995)^{2}$, menciona diversas entidades abocadas a esta tarea en América Latina y en resto globo

1 Se llamará de manera indistinta Mont Pèlerin Society o Sociedad a lo largo de escrito para hacer referencia a tal asociación.

2 Escrita por uno de los que fuese su miembro y presidente, Max Hartwell. 
durante las décadas de 1950 y 1960. Entre tales entidades y para el caso de Argentina, cita precisamente al Centro de Difusión de la Economía Libre (CDEL) y a la figura de su presidente y fundador, Alberto Benegas Lynch. El CDEL si bien reproducirá escritos de diversas escuelas insertas en el movimiento neoliberal focalizará su interés en la escuela austríaca de economía (Büren de, 2014).

Si bien Friedrich Hayek, uno de los más conocidos representantes de la escuela austríaca de economía, es quien encabeza la fundación de la Mont Pèlerin Society, este espacio está conformado en sus inicios por una pluralidad de perspectivas entre las que se encuentran, además de la mencionada: la escuela de Chicago, el libertarianismo, la economía social de mercado y la escuela de Virginia. A partir de esto, es importante destacar que al tiempo que las inserciones del pensamiento montpelerinés de los años 50 y 60 en América Latina se realizan mayoritariamente de forma directa a través de la escuela austríaca de economía, las inserciones en dicho territorio en periodos más recientes del siglo XX se realizarán mayoritariamente de forma mediatizada a través de la escuela de Chicago. Esto permite comprender por qué los estudios que trabajan la penetración del neoliberalismo en América Latina en los años 70 hacen alusión al monetarismo de la escuela de Chicago liderada por Milton Friedman y por qué el presente trabajo retoma una corriente menos visibilizada que desarrolla sus arribos desde una época anterior (Büren de, 2013a).

Entonces, entendiendo la labor de los intelectuales orgánicos y las construcciones discursivas que estos desarrollan y difunden como uno de los elementos de los cuales las fracciones de clase dominante se valen para alimentar su dirección hegemónica (Gramsci, 2009), analizamos algunas prácticas locales en Argentina destinadas a articular sus acciones con este movimiento de escala mundial, así como las vinculaciones del mismo con empresarios, militares y políticos locales. Más precisamente, analizaremos una fracción local de tal movimiento, un sector más estrechamente vinculado con la corriente austríaca del movimiento y encabezado por la figura de Alberto Benegas Lynch y las instituciones fundadas por él y su familia ${ }^{3}$. En

3 Este artículo focaliza su atención en el despliegue de fuentes documentales que permiten dar cuenta de los resultados empíricos de la investigación que aquí se presen- 
términos amplios, y con vistas que superan este escrito, la hipótesis general que sigue este trabajo es aquella que formulase Antonio Gramsci en sus escritos respecto de la existencia de una fuerte articulación entre intelectuales y sectores dominantes en la construcción del gobierno hegemónico. De manera más concreta, nos planteamos como hipótesis específica de este trabajo la existencia de fuertes articulaciones entre intelectuales productores y difusores discursivos vinculados a la Mont Pèlerín Society y sectores dominantes del ámbito local argentino, es decir, particularmente, a partir del análisis de fuentes documentales cuya autoría les pertenece, buscamos observar el modo en el que se despliega la estrategia monpelerinesa en Argentina en un periodo concreto.

\section{Desarrollo}

1. Alberto Benegas Lynch: entre el derrocamiento del peronismo y el ingreso del proyecto neoliberal

Compartimos con el lector, antes de iniciar el escrito, fragmentos del homenaje del periódico La Nación -espacio de expresión de sectores de raigambre patricio en Argentina- a Alberto Benegas Lynch padre en el día de su fallecimiento. La siguiente cita permite observar lo que Foucault (2007b) denomina como la "posición de sujeto" desde la que Benegas Lynch se pronuncia, su emplazamiento de clase, su rango en la estructura empresarial, política e intelectual nacional, es decir, nos permite visibilizar que su figura no se reduce a aquello que Gramsci (2008) define como un "intelectual orgánico" -clase subsidiaria de sectores dominantes-, sino que se constituye ella misma en miembro y representante de fracciones dominantes de clase locales y en un actor central de articulación entre el patriciado vernáculo y el empresariado internacional:

Causó gran pesar en los ámbitos económico y académico el fallecimiento, ayer, del doctor Alberto Benegas Lynch (...) Hombre de

ta. Por cuestiones de extensión, los abordajes teóricos que delimitan este trabajo empírico y las discusiones teóricas a las que da lugar sólo se mencionan brevemente en el presente manuscrito; sin embargo, han sido desarrollados de manera profusa por la autora en otros escritos (de Büren, 2013b, 2014, 2015b, 2017, 2018, 2019, 2020). El lector interesado en tal profundización, deberá remitirse a los artículos aquí referenciados. 
bien (...) Durante más de cuarenta años presidió la empresa familiar fundada por su abuelo en 1883, en el área de la vitivinicultura. Llegó a ser presidente de la Asociación Vitivinícola Argentina y de la Cámara Argentina de Comercio. (...) [A]cadémico titular de la Academia Nacional de Ciencias Económicas y de la Academia Nacional de Ciencias Morales y Políticas (...) [y] de la Academia Nacional de Economía del Uruguay.

En 1949 dio el primer paso para el establecimiento del Centro de Estudios sobre la Libertad (...) [donde] ofreció decenas de becas para que jóvenes profesionales pudieran estudiar en los Estados Unidos (...) [Quienes] iniciaron en el país el debate sobre las ventajas de una economía en la que los consumidores pueden elegir los servicios de su agrado, sin injerencias gubernamentales.

Durante los dos primeros años de la Revolución Libertadora vivió en Washington, donde fue ministro plenipotenciario de la embajada argentina. (...) [S]iendo titular del Encuentro Nacional Republicano, unió fuerzas cívicas de centro en distintos momentos históricos para contener los avances estatistas. (La Nación, 1999, párr. 1-8).

Alberto Benegas Lynch conoció al dirigente y fundador de la Mont Pèlerin Society, Friedrich von Hayek, en el año 1950, es decir, a tres años de fundada la Sociedad (1947) y a dos de ingresado von Hayek a la Universidad de Chicago (1948). En 1957 - al cabo de 7 años de dicho evento, a 2 años del derrocamiento y proscripción del peronismo y tras el bombardeo de Plaza de Mayo el 16 de junio de 1955- Friedrich von Hayek visita la Argentina invitado por el Centro de Difusión de la Economía -institución fundada por Alberto Benegas Lynch específicamente para la difusión en el espacio local del pensamiento montpelerines- y se reúne con el entonces presidente de facto, el teniente General Pedro Eugenio Aramburu (Hartwell, 1995; Benegas Lynch, 1978).

Alberto Benegas Lynch junto a Joaquín Reig y Gustavo Velasco publican en 1961 el texto Deliberaciones Sobre la Libertad. El primero de ellos obsequia un ejemplar en papel en julio de 1961 al teniente general Carlos Severo Toranzo Montero con la dedicatoria escrita con su puño y letra que compartimos, a continuación, con el lector: 


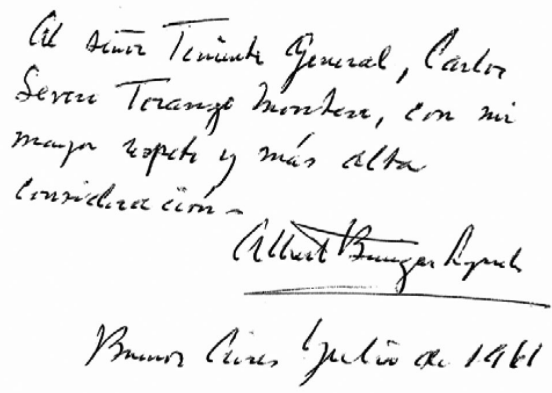

Recordemos que Carlos Severo Toranzo Montero se desempeñó como comandante en jefe de ejército argentino durante la presidencia de Arturo Frondizi (1958-1962); dirigió sus influencias a la puesta en uso del plan CONINTES (Conmoción de Orden Interno), intentó de forma fallida- promover la pena de muerte mediante la aplicación de la ley marcial como forma de combatir las protestas obreras, entre ellas, las huelgas de trabajadores ferroviarios; fue miembro de la fracción colorada del ejército; dirigió su poder a reprimir la acción obrera, los movimientos marxistas y peronistas. Respecto de los últimos, consideraba, había que evitar que tendieran hacia la "izquierda castrista"

En articulación con ello, en tal superficie histórica, en el año 1961, el Centro de Difusión de la Economía Libre reproduce en idioma local el "Capítulo XVIII" del trabajo de von Hayek Fundamentos de la libertad de 1961 (2008) bajo la denominación Los sindicatos y la ocupación obrera (1961). Este escrito coloca a la criminalización de las acciones de protesta sindical como parte integrante de estado de derecho, una forma estatal defendida por ellos en tanto, afirman, garante de la libertad individual (Büren de, 2020).

\section{Disputas locales en el campo del saber económico experto}

El Centro de Difusión de la Economía Libre, que Benegas Lynch fundó y dirigió, invitó a disertar en Argentina a numerosos intelectuales de la Mont Pèlerin Society. En junio de 1959 -al año siguiente de que la Universidad de Buenos Aires inaugurara el dictado de una de las primeras

4 En relación con la disputa entre azules y colorados en el ejército, se puede consultar Rouquié (1982) y en relación con el plan CONINTES, véase Belenky (1984) y Novaro (2010). 
licenciaturas en economía política del país- fue el turno de Ludwig von Mises. Su exposición se llevó a cabo en el auditorio de la Facultad de Ciencias Económicas de la mencionada universidad, fue dirigida hacia el público en general y contó con la presencia de empresarios, exfuncionarios -como Federico Pinedo y el exministro de comercio de la revolución libertadora, Juan Llamazares- y estudiantes de la licenciatura en economía (Mises von, 1959).

Margit von Mises (1996), su esposa, describe tal episodio:

A finales de 1958, mi marido recibió la amable invitación del doctor don Alberto Benegas Lynch para ir (...) a Buenos Aires y dictar allí una serie de conferencias (...). Llegamos a Argentina algunos meses después del derrocamiento de Perón. Había destrozado el país. (...) Ludwig von Mises habló (...) a favor del capitalismo; criticó el socialismo y el intervencionismo, el comunismo y el fascismo. (p.277).

Esta descripción permite observar el cariz político del desembarco de este miembro fundador de la Mont Pèlerin Society en tierras argentinas en el contexto general de la estrategia desplegada por la asociación.

Las invitaciones del Centro de Difusión de la Economía Libre (CDEL) -después rebautizado como Centro de Estudios sobre la Libertad- a integrantes de la Mont Pèlerin Society se siguieron sucediendo, pero es de destacar que las dos visitas mencionadas, así como los inicios de su publicación periódica Ideas sobre la Libertad en diciembre de 1958 (Ideas sobre la Libertad, 1958), fueron efectuadas al calor de la creación de las primeras licenciaturas en economía de Argentina y de los inicios de la década (1955-1965) que Manuel Fernández López (2001) denomina "la edad de oro de los economistas" (p. 17). Fue así bautizada no sólo por la creación de la carrera universitaria de la licenciatura en economía, sino también por: los importantes avances en el bienio 1957-1958 sobre una teoría de la inflación de desarrollo puramente nacional: la teoría no monetaria de la inflación de Julio Olivera; la fundación de la Asociación Argentina de Economía Política (AAEP) en 1957; el surgimiento de institutos -como el Instituto de Desarrollo Económico y Social (IDES) en 1960; y la emergencia de publicaciones -como la Revista de Desarrollo Económico que lanza su primer ejem- 
plar en febrero de 1959-, dedicadas al estudio de la economía local ${ }^{5}$. Esto no constituye un dato menor si se tiene presente la función que la Mont Pèlerin Society otorga al discurso y a las instituciones científicas en la influencia y dominio del hacer político, económico y social, y si se considera que tal apogeo de la investigación económica y la producción científica local cierra su ciclo con la dictadura de Onganía y la expulsión de científicos locales.

\section{ESEADE en la bisagra. Entre los procesos de industrializa- ción sustitutiva de importaciones y la acumulación financiera}

Una de las características centrales del autodenominado proceso de reorganización nacional, que se extendió en Argentina entre 1976 y 1983, fue el intenso despliegue y aplicación de políticas económicas de corte monetarista. Tales medidas dieron como resultado la conclusión del proyecto de industrialización sustitutivo de importaciones y el inicio de un proceso de acumulación que ya no sería guiado por la producción y el empleo industrial, sino por la valorización financiera. Esto implicó una profunda transformación en la estructura económica y social nacional (Basualdo, 2006; Pucciarelli, 2004; Schorr, 2006). A partir de este momento, los diversos sectores económicos subordinaran sus esfuerzos y beneficios a la preeminencia del sector financiero (Vuolo lo, 2000), mientras que el empleo marginal, el empleo informal y la desocupación irán en crecimiento y se constituirán en las principales variables de ajuste económico (Torrado, 1994, 2006).

Esta superficie histórica, esto es, la Argentina de fines de la década de 1970, es el momento en el cual las políticas neoliberales de corte monetaristas efectivizan su ingreso sin ninguna reticencia debido al silenciamiento de las resistencias obreras. La Fundación Bolsa de Comercio de Buenos Aires y el CDEL de manera conjunta lanzan va-

5 Para un estudio más detallado de la historia del desarrollo institucional saber económico a lo largo del siglo XX, sobre todo para el análisis de su institucionalización en el ámbito universitario, se recomienda Fernández López (2001, 2008), Neiburg y Plotkin (2004), Rozenwurcel, Bezchinsky y Chatruc Rodríguez (2007), y Unzué (2020). Para el análisis del pensamiento económico hegemónico en el espacio argentino en las tres últimas décadas del siglo XX, se recomienda Morresi (2008), Heredia (2004, 2006), Beltrán (2005a, 2005b), Roig (2007), Grondona $(2011,2012)$ y Rubinich (2001). Para el análisis de las corrientes teóricas económicas en discusión protagónica en el espacio argentino y latinoamericano en el siglo XX, ver Sztulwark (2003), Castellani (2006) y Astarita (2010). 
rias publicaciones de obras de autores austríacos, tanto de origen local como foráneo -entre ellos, Alberto Benegas Lynch, Ludwig von Mises, Friedrich von Hayek, Murray Rothbart y Hans Sennholz ${ }^{6-}$ e invitan a Friedrich von Hayek a brindar una serie de conferencias en las instalaciones de la Bolsa de Comercio en noviembre de 1997.

En sinfonía con este proceso, y tal como ya hiciese Alberto Benegas Lynch a fines de los años 1950 mediante la fundación del CDEL, su primogénito -quien detenta igual nombre- crea la Escuela Superior de Economía y Administración de Empresas (ESEADE) con idéntico objetivo en 1978.

Alberto Benegas Lynch hijo anuncia la inauguración de esta institución y solicita apoyo financiero en la Bolsa de Comercio de Buenos Aires en presencia de, entre otros, Manuel Ayau y Federico Zorraquín. Manuel Ayau se desempeñó como presidente de la Mont Pèlerin Society entre 1978 y 1980 y como rector de la Universidad Francisco de Marroquín (Guatemala) ${ }^{7}$. Federico Zorraquín ha sido dueño de uno de los holdings más grandes de Argentina, dirigente de la Asociación de Bancos Argentinos (ADEBA), representante del Consejo Empresario de América Latina, director de la Fundación de Investigaciones Económicas Latinoamericanas (FIEL) y autoridad en el Consejo Empresario Argentino (CEA); ha recibido de manos del ESEADE -institución que él mismo financia- un doctorado honoris causa en el año 2008 y ha sido uno de los principales beneficiarios de fondos del Banco Nación en el último periodo dictatorial en Argentina (Basualdo, Santarcángelo, Wainer, Russo \& Perrone, 2016; La Nación, 23/11/2007).

Así relata Alberto Benegas Lynch hijo; quien pusiera en funcionamiento la ESEADE y la dirigiera en calidad de rector entre 1978 y 2001 ; la historia de la formación de dicha institución, sus objetivos, sus fuentes de financiamiento, sus miembros fundadores y su vinculación con integrantes de la Mont Pèlerin Society:

6 Entre tantos otros, podemos citar los siguientes, cuya referencia completa se detalla en las referencias: von Mises (1978, 1979), von Hayek (1978), Rothbard (1979); Benegas Lynch, García Belsunce, Loncán y Luzzetti (1979); Sennholz (1977) y Reig Albiol y Sennholz (1977).

7 Institución orientada a contrarrestar la influencia socialista en América Latina. Puede visitar su página oficial en https://www.ufm.edu/index.php/En ese pórtal se puede encontrar el desarrollo de su historia, objetivos, miembros y vínculos con otras instituciones. 
En el contexto de marcos democráticos (...) quienes apuntan a ejercer el poder lo deben realizar con un discurso que resulte aceptable para la opinión pública (...), a su vez, la opinión dominante se alimenta de las ideas que mayor peso y difusión. (Benegas Lynch, 2007, párr. 3).

Sobre esta base y estas inquietudes se constituyó la institución de posgrado ESEADE. (...) [T]rabajé en lo que sería la primera Maestría independiente que se dictaría en la Argentina (...) dirigida al mundo de los negocios pero con una carga académica suficiente en el campo de la economía, el derecho y la filosofía, al efecto de que al futuro dirigente empresario (...) se le ofrecieran elementos adecuados para la comprensión del contexto en el que se desenvuelve su empresa. (Benegas Lynch, 2007, párr. 5).

Durante todo el año 1977 el que suscribe destinó una parte sustancial de su tiempo (...) ante la comunidad empresaria al efecto de lograr la financiación (...). Finalmente se logró el objetivo a principios de 1978 gracias a (...) un grupo de empresarios (...) [C]onsidero de especial relevancia detenerme en dos nombres que representan (...) a tantas personas que desde tan diversos orígenes empresarios y académicos hicieron posible la existencia de esta casa de estudios. Se trata de Federico Zorraquín y de Friederich A. Hayek. (...) Zorraquín financió ESEADE (...). Al profesor Hayek (...) lo visité (...) para sugerirle que presidiera el (...) Consejo Consultivo. (Benegas Lynch, 2007, párr. 8).

Esta declaración nos permite, observar el modo en que las formas de sustentación del capitalismo como modo de ordenamiento y jerarquización social se actualizan, vuelven una y otra vez a reeditar la construcción de sus bases de legitimación.

Más ampliamente, entendemos que tras la caída de las formas de gobierno monárquico -cuyo poder descansaba en gran medida en la estructura y el discurso religioso- y el advenimiento de las democracias parlamentarias, se torna necesaria la elaboración y difusión de un discurso que garantice el mantenimiento y la perpetuación de los privilegios de clase en los cuales el capitalismo se asienta. En concordancia con ello, los intelectuales, empresarios e instituciones vinculados a la Mont Pèlerin Society entienden que la influencia en el quehacer 
público y la conformación social no se consigue perpetrar exclusivamente a través de la injerencia directa en el Estado o de la política partidista sino, fundamentalmente, mediante la influencia en los actores que participan en tales espacios y en la opinión pública general. Esto vuelve imperante el financiamiento de instituciones que detentan el poder de enunciar aquella palabra -aquella forma discursiva- que ha conquistado una mayor fuerza de verdad desde el iluminismo hasta la actualidad en el mundo occidental: el saber científico o, más recientemente, el saber experto.

A las personalidades aludidas en la declaración del rector de la ESEADE se agregan, según permite observar la sección institucional de su página de internet oficial, otros empresarios financistas fundadores:

La antes referida Asamblea de Fundadores que brindó el primer apoyo financiero a ESEADE y posibilitó su funcionamiento, estuvo originalmente integrada por Abel Ayerza, Armando M. Braun, Federico Carlés, Rodolfo Costantini, Miguel de los Santos, Enrique Duahu, José A. Estenssoro, Jose A. Esteves, Amalia Lacroze de Fortabat, Roberto Helguera, Guillermo M. Lovegrove, Alfredo D. Olaechea, José E. Rohm, Oscar Secco, Alberto Servente, Mario Vásquez, Wilfred von Büllow, Guillermo Yeatts y Federico L. Zorraquín, algunos de los cuales integraron el primer Consejo Directivo juntamente con el suscripto, quienes también continuaron con la financiación y estímulo moral a través del tiempo. (Benegas Lynch, 2007, párr. 14).

Se señalan, además, empresarios y personajes públicos que han integrado el Consejo Directivo:

El Consejo Directivo (...) se ha conformado por importantes personalidades empresarias, emprendedores sociales y personalidades públicas. Actualmente se encuentra presidido por Enrique Duhau (h), y conformado por Santiago Bergadá Moritán, Gerardo Bongiovanni, Eduardo Bruchou, Emilio Cárdenas, Clarisa Estol, Alejandro Estrada, Mariano Grondona, Alfredo Irigoin, Eduardo Marty, Luisa Zorraquín y Enrique Zuleta Puceiro. (Gómez, 2012, párr. 1).

Y docentes que dictaron allí sus cátedras:

Entre ellos se puede mencionar a Juan Carlos Alonso, Juan Anich, Juan Carlos Cachanosky, Roberto Cortés Conde, Jorge del Águila, Ezequiel Gallo, Jorge García Venturini, Juan José Gilli, Enrique Lon- 
can, Carlos Alberto Loprete, Isidoro Marín, Jorge Mocetti, Eduardo O'Connor, Armando Ribas, Marco Aurelio Risolía, Salvador Ruggeri, Esteban Thomsen y Gabriel Zanotti. (Gómez, 2012, párr. 2).

Alberto Benegas Lynch padre será desde 1957 miembro integrante de la Mont Pèlerin Society (Benegas Lynch, 1997), con acceso en calidad de expositor a los general meetings desde al menos el año 1961. Momento, este último, en el cual el primo de Ernesto "Che" Guevara Lynch de la Serna -quien no ha escatimado agravio a la hora de criticar el accionar político de su familiar- participa de la $12^{\mathrm{a}}$ meeting desarrollado en Turín, Italia, entre el 3 y 9 de septiembre con la ponencia "Communism in Latin America from an argentine point of view and with reference to the notion of underdeveloped countries" (Liberaal Archief, 2004) ${ }^{8}$. Desde su inserción en la Sociedad, la participación argentina en esta irá en crecimiento. A continuación, apreciaremos algunos datos censales al respecto.

\section{Participación argentina y latinoamericana en Mont Pèlerin Society}

El cuadro 1 permite observar el origen geográfico de los miembros de la Mont Pèlerin Society en el transcurso de tiempo de 1947 a 1989. En dicho lapso, el número de miembros totales de la sociedad asciende desde 103 hasta 489 y la cantidad de miembros argentinos se eleva de 1 a 11. En otros términos, la cifra de argentinos monpelerineses crece a un ritmo similar al del total de la Sociedad, lo que hace que la relevancia relativa de aquellos en su composición se mantenga estable y asuma a lo largo del periodo valores que oscilan entre el 0,5\% y 2,2\%. Si se analiza la participación continental, se observa que la importancia relativa en América Latina y Central crece: pasa de conformar el 1\% del total de los miembros en 1947-1949 a alcanzar el 9,4\% de los mismos en 1989. También crece la afiliación en América del Norte -traccionada por la participación estadounidense- que pasa del 37,9\% al 47,9\% en igual periodo, con un pico en 1981 cuando consigue a detentar la mitad, el 50,1\%, de los miembros. Por otro lado, Asia -impulsada por Japón- eleva su participación del 0,1\% al 7,4\%, como también lo hace

8 La Mont Pèlerin Society, efectúa tres tipos de reuniones: Generales, convocadas en los países centrales y de las cuales participan figuras líderes; Regionales, desarrolladas en países periféricos con la asistencia de personajes de menor centralidad y Especiales. 
Oceanía que aumenta del $0 \%$ al 2,86\%. Si todos los continentes hasta aquí mencionados ganan espacio en la Mont Pèlerin Society, ¿qué bloque territorial empobrece su relevancia relativa? Europa retrocede no sólo en términos de participación absoluta y relativa de sus nativos, sino también en relación a la preeminencia de sus consideraciones teórico-políticas. Ello es particularmente intenso en el caso de los adherentes a la economía social de mercado, una perspectiva que progresivamente pierde relevancia y capacidad de decisión al interior de la Sociedad y en el delineamiento del arte de gobierno neoliberal que tal asociación pretende fundar, difundir e implementar. En consecuencia, es comprensible el hecho de que los arribos del neoliberalismo efectivizados en el último cuarto del siglo XX en Argentina y Chile, los cuales han detentado mayor reconocimiento público, hayan sido concretados desde la ciudad, la Universidad y la Escuela de Chicago.

Otro elemento que distingue la información procesada por François Denord que presentamos en el cuadro 1 es la relevancia dentro del conglomerado latino y centroamericano de la participación de Argentina y Guatemala en la Sociedad. Punto en el cual volvemos a recalcar que el guatemalteco Manuel Ayau, quien fundó la Universidad Francisco de Marroquín y quien vino a la presentación de la ESEADE, fue el único latinoamericano que ejerció alguna vez la presidencia de la sociedad en el transcurso del siglo XX. Lo hizo precisamente entre 1978 y 1980 (Liberal Archief, 1998). 
Cuadro 1

Orígenes geográficos de los miembros de la Mont Pèlerin Society (1947-1989) ${ }^{9}$

\begin{tabular}{|c|c|c|c|c|c|c|}
\hline País de residencia & $\begin{array}{c}1947- \\
1949 \\
(\mathrm{n}=103)\end{array}$ & $\begin{array}{c}1956 \\
(n=204)\end{array}$ & $\begin{array}{c}1963 \\
(n=295)\end{array}$ & $\begin{array}{c}1973 \\
(n=377)\end{array}$ & $\begin{array}{c}1981 \\
(n=414)\end{array}$ & $\begin{array}{c}1989 \\
(n=489)\end{array}$ \\
\hline África del Sur & $0,0 \%$ & $2,0 \%$ & $2,0 \%$ & $0,3 \%$ & $1,0 \%$ & $1,6 \%$ \\
\hline Argentina & $1,0 \%$ & $0,5 \%$ & $2,0 \%$ & $1,9 \%$ & $2,2 \%$ & $2,2 \%$ \\
\hline Guatemala & $0 \%$ & $0,0 \%$ & $0,0 \%$ & $0,5 \%$ & $1,7 \%$ & $1,4 \%$ \\
\hline $\begin{array}{l}\text { América Central y } \\
\text { Latina }\end{array}$ & $1,0 \%$ & $3,4 \%$ & $5,8 \%$ & $8,0 \%$ & $8,9 \%$ & $9,4 \%$ \\
\hline Estados Unidos & $37,9 \%$ & $36,8 \%$ & $39,7 \%$ & $44,8 \%$ & $49,5 \%$ & $46,2 \%$ \\
\hline América del Norte & $37,9 \%$ & $37,3 \%$ & $40,1 \%$ & $45,4 \%$ & $50,1 \%$ & $47,9 \%$ \\
\hline Japón & $0,1 \%$ & $0,1 \%$ & $1,7 \%$ & $4,2 \%$ & $4,6 \%$ & $4,9 \%$ \\
\hline Asia & $0,1 \%$ & $0,5 \%$ & $2,0 \%$ & $5,3 \%$ & $5,6 \%$ & $7,4 \%$ \\
\hline Francia & $15,5 \%$ & $10,3 \%$ & $9,8 \%$ & $7,7 \%$ & $5,1 \%$ & $3,9 \%$ \\
\hline Gran Bretaña & $14,6 \%$ & $14,2 \%$ & $9,5 \%$ & $9,0 \%$ & $8,0 \%$ & $6,5 \%$ \\
\hline Italia & $3,9 \%$ & $5,4 \%$ & $3,4 \%$ & $2,1 \%$ & $2,4 \%$ & $2,0 \%$ \\
\hline $\begin{array}{l}\text { República Federal } \\
\text { Alemana }\end{array}$ & $9,7 \%$ & $8,3 \%$ & $11,9 \%$ & $11,9 \%$ & $8,7 \%$ & $7,4 \%$ \\
\hline Suiza & $6,8 \%$ & $5,9 \%$ & $3,4 \%$ & $2,1 \%$ & $2,2 \%$ & $2,2 \%$ \\
\hline Europa & $61,2 \%$ & $55,4 \%$ & $49,8 \%$ & $40,8 \%$ & $33,1 \%$ & $30,9 \%$ \\
\hline Australia & $0,0 \%$ & $0,5 \%$ & $0,0 \%$ & $0,3 \%$ & $1,4 \%$ & $2,7 \%$ \\
\hline Oceanía & $0,0 \%$ & $0,98 \%$ & $0,34 \%$ & $0,3 \%$ & $1,4 \%$ & 2,86 \\
\hline
\end{tabular}

Fuente: Denord (2002, p. 18).

El cuadro 2 permite observar que América Latina ha sido elegida sede de la General Meetings sólo una vez, en el año 2006, en la tierra del ex-presidente montpelerinés Manuel Ayau: Guatemala. Esta porción del continente fue sede de reuniones regionales en ocho oportunidades: Caracas (Venezuela) en 1969; Guatemala (Guatemala) en 1973; Viña del Mar (Chile) en 1981; Antigua (Guatemala) en 1990; Río de Janeiro (Brasil) en 1993; Cancún (México) en 1996 y Buenos Aires (Argentina) en 2011 y fue sede de reuniones especiales una vez, en Galápagos (Ecuador) en 2013.

9 El cuadro solo releva los países que contribuyen en mayor porcentaje al reclutamiento de la Sociedad Mont Pèlerin. 
Cuadro 2

Reuniones de la Mont Pèlerin Society

\begin{tabular}{|c|c|c|c|c|c|c|}
\hline \multicolumn{7}{|c|}{ Reunión fundacional: Mont Pèlerin, Suiza, 1947} \\
\hline \multicolumn{2}{|c|}{ Reuniones generales } & \multicolumn{2}{|c|}{ Reuniones regionales } & \multicolumn{3}{|c|}{ Reuniones especiales } \\
\hline Ciudad & País & Año Ciudad & País & Año Ciudad & País & Año \\
\hline Seelisberg & Suiza & 1949 Tokyo & Japón & 1966 Taipei & Taiwan & 1978 \\
\hline Bloemendaal & Holanda & 1950 Caracas & Venezuela & 1969 Taipei & Taiwan & 1988 \\
\hline Beauvallon & Francia & 1951 Rockford & EEUU & $1971 \begin{array}{l}\text { Mont } \\
\text { Pelerin }\end{array}$ & Suiza & 1997 \\
\hline Seelisberg & Suiza & 1953 Salzburg & Austria & 1973 Bali & Indonesia & 1999 \\
\hline Venecia & Italia & 1954 Guatemala & Guatemala & 1973 Goa & India & 2002 \\
\hline Berlin & Alemania & 1956 Hillsdale & EEUU & 1975 Sri Lanka & SriLanka & 2004 \\
\hline St. Moritz & Suiza & 1957 Paris & Francia & 1977 Nairobi & Kenya & 2007 \\
\hline Princeton & EEUU & 1958 Amsterdam & Holanda & 1977 New York & EEUU & 2009 \\
\hline Oxford & Inglaterra & 1959 Madrid & España & 1979 Estambul & Turquía & 2011 \\
\hline Kassel & Alemania & 1960 Stockholm & Suecia & $1981 \mathrm{Fez}$ & Marruecos & 2012 \\
\hline Turín & Italia & 1961 Viña del Mar & Chile & 1981 Galápagos & Ecuador & 2013 \\
\hline Knokke & Bélgica & 1962 Vancouver & Canada & 1983 & & \\
\hline Semmering & Austria & 1964 Paris & Francia & 1984 & & \\
\hline Stresa & Italia & 1965 Sydney & Australia & 1985 & & \\
\hline Vichy & Francia & 1967 Indianapolis & EEUU & 1987 & & \\
\hline Aviemore & Escocia & 1968 Christchurch & Nueva Zelanda & 1989 & & \\
\hline Munich & Alemania & 1970 Antigua & Guatemala & 1990 & & \\
\hline Montreux & Suiza & $\begin{array}{ll}1972 & \begin{array}{l}\text { Big Sky, } \\
\text { Montana }\end{array} \\
\end{array}$ & EEUU & 1991 & & \\
\hline Bruselas & Bélgica & 1974 Praga & Checoslovaquia & 1991 & & \\
\hline St. Andrews & Escocia & $1976 \begin{array}{l}\text { Rio de } \\
\text { Janeiro }\end{array}$ & Brasil & 1993 & & \\
\hline Hong Kong & Hong Kong & 1978 Cape Town & Sudáfrica & 1995 & & \\
\hline Stanford & EEUU & 1980 Cancún & México & 1996 & & \\
\hline Berlin & Alemania & 1982 Barcelona & España & 1997 & & \\
\hline Cambridge & Inglaterra & 1984 Vancouver & Canadá & 1999 & & \\
\hline St. Vincent & Italia & 1986 Potsdam & Alemania & 1999 & & \\
\hline Tokyo & Japón & 1988 Bratislava & Eslovaquia & 2001 & & \\
\hline Munich & Alemania & 1990 Chattanooga & EEUU & 2003 & & \\
\hline Vancouver & Canada & 1992 Hamburg & Alemania & 2004 & & \\
\hline Cannes & Francia & 1994 Reykjavik & Iceland & 2005 & & \\
\hline Vienna & Austria & 1996 Stockholm & Suecia & 2009 & & \\
\hline $\begin{array}{l}\text { Washington } \\
\text { D.C. }\end{array}$ & EEUU & 1998 Buenos Aires & Argentina & 2011 & & \\
\hline Santiago & Chile & 2000 New Delhi & India & 2011 & & \\
\hline London & Inglaterra & 2002 & & & & \\
\hline Salt Lake City & EEUU & 2004 & & & & \\
\hline Guatemala & Guatemala & 2006 & & & & \\
\hline Tokyo & Japón & 2008 & & & & \\
\hline Sydney & Australia & 2010 & & & & \\
\hline Praga & $\begin{array}{l}\text { República } \\
\text { Checa }\end{array}$ & 2012 & & & & \\
\hline Hong Kong & China & 2014 & & & & \\
\hline
\end{tabular}

Fuentes: Mont Pèlerin Society (2013a) y Mont Pèlerin Society (2013b). 
En el año 2011, la Sociedad eligió como lugar de reunión regional la Ciudad Autónoma de Buenos Aires y organizó dicho evento a través de la labor de la Fundación Libertad ${ }^{10}$ y lo denomínó "The Mont Pèlerin Society Regional Meeting-Buenos Aires: the populist challenge to Latin American liberty". El cuadro 3 que presentamos a continuación condensa los disertantes del evento, entre quienes se destacan: políticos, como el expresidente de Argentina Mauricio Macri, el jefe de gabinete del gobierno chileno Cristián Larroulet y el expresidente de Bolivia Jorge Quiroga; ex ministros de economía latinoamericanos, como Carlos Cáceres, quien dirigió el ministerio durante la última dictadura chilena, y Manuel Hinds, quien se desempeñó como ministro de economía de El Salvador; ensayistas, como Mario Vargas Llosa, Marcos Aguinis y Carlos Alberto Montaner; reconocidos economistas argentinos y extranjeros, como Ricardo López Murphy, Jorge Ávila, Roberto Salinas León (México) y Sebastián Edwards (Chile); historiadores, como Roberto Cortés Conde y Ezequiel Gallo; adherentes locales a la escuela austríaca de economía y habituales columnistas del diario La Nación como, Alberto Benegas Lynch, Martín Krause y Gabriel Zanotti; extranjeros como Enrique Ghersi (Perú); profesores, miembros directivos de universidades y de centros relacionados con la Mont Pèlerin Society, como Atlas Economic Research Foundation (Estados Unidos), Heritage Foundation, Junior Achievement, Cato Institute (Estados Unidos), Fundación Libertad, Fundación Atlas 1853, The Manhattan Institute, Liberty Fund, Instituto Libertad y Desarrollo (Chile), Free Market Research Foundation, The Ayn Rand Institute, Universidad Católica del Perú, Universidad de Los Andes (Chile), Universidad Francisco Marroquín (Guatemala), ESEADE, Centro de Estudios Públicos (Chile), Centro de Estudios Macroeconómicos de Argentina (CEMA), Universidad de Chicago, Universidad Adolfo Ibáñez (Chile).

10 La Fundación Libertad tiene su dirección física en la localidad de Rosario ( Argentina) y sus actividades pueden conocerse en su página web oficial: http://www.libertad. org.ar/ 
Cuadro 3

Disertantes de The Mont Pèlerin Society Regional Meeting. Buenos Aires. 2011.

\begin{tabular}{|c|c|c|}
\hline Participante & Procedencia institucional & $\begin{array}{c}\text { País de } \\
\text { procedencia } \\
\text { institucional }\end{array}$ \\
\hline $\begin{array}{l}\text { Alberto Benegas } \\
\text { Lynch (hijo) }\end{array}$ & $\begin{array}{l}\text { Economista, ex vicepresidente de MPS; } \\
\text { Presidente de la sección económica de la } \\
\text { Academia Nacional de Ciencias }\end{array}$ & Argentina \\
\hline Alejandro Chafuen & $\begin{array}{l}\text { Presidente de Atlas Economic Research } \\
\text { Foundation }\end{array}$ & Estados Unidos \\
\hline Alfredo Bullard & $\begin{array}{l}\text { Profesor de derecho civil y análisis económico } \\
\text { del derecho de la Pontificia Universidad } \\
\text { Católica del Perú }\end{array}$ & Perú \\
\hline Alvaro Vargas Llosa & $\begin{array}{l}\text { Miembro principal del The Center on Global } \\
\text { Prosperity at the Independent Institute }\end{array}$ & Estados Unidos \\
\hline Andrei Illarionov & $\begin{array}{l}\text { Miembro principal del Center for Global } \\
\text { Liberty and Prosperity del Cato Institute }\end{array}$ & Estados Unidos \\
\hline Angel Soto & Profesor, Universidad de Los Andes & Chile \\
\hline $\begin{array}{l}\text { Augusto } \\
\text { Zimmermann }\end{array}$ & $\begin{array}{l}\text { Asociación dean de la Universidad Murdoch, } \\
\text { Escuela de Derecho }\end{array}$ & Brasil \\
\hline $\begin{array}{l}\text { Carlos Alberto } \\
\text { Montaner }\end{array}$ & Escritor & Cuba \\
\hline Carlos Cáceres & $\begin{array}{l}\text { Economista, ministro de economía durante la } \\
\text { última dictadura chilena }\end{array}$ & Chile \\
\hline $\begin{array}{l}\text { Carlos Rodríguez } \\
\text { Braun }\end{array}$ & $\begin{array}{l}\text { Profesor de la Universidad Complutense de } \\
\text { Madrid }\end{array}$ & España \\
\hline Carlos Sabino & $\begin{array}{l}\text { Historiador económico, Universidad } \\
\text { Francisco Marroquín }\end{array}$ & Guatemala \\
\hline Carola Pessino & $\begin{array}{l}\text { Profesora de economía, Universidad Torcuato } \\
\text { Di Tella }\end{array}$ & Argentina \\
\hline Claudio Véliz & Historiador, sociólogo y autor & Chile \\
\hline Cristian Larroulet & $\begin{array}{l}\text { Jefe del gabinete de ministros, gobierno de } \\
\text { Chile }\end{array}$ & Chile \\
\hline $\begin{array}{l}\text { Darío Fernández } \\
\text { Morera }\end{array}$ & Exministro de economía & Estados Unidos \\
\hline Deepak Lal & $\begin{array}{l}\text { Profesor de estudios de desarrollo } \\
\text { internacional de la Universidad de California. } \\
\text { Vicepresidente senior de la Mont Pèlerin } \\
\text { Society }\end{array}$ & India \\
\hline Ed Feulner & $\begin{array}{l}\text { Expresidente de la Mont Pelerin Society, } \\
\text { presidente de Heritage Foundation }\end{array}$ & Estados Unidos \\
\hline Eduardo Marty & $\begin{array}{l}\text { Director general, Junior Achievement } \\
\text { Argentina }\end{array}$ & Argentina \\
\hline Enrique Ghersi & Abogado, profesor de la Universidad de Lima & Perú \\
\hline Ezequiel Gallo & Dr. en Historia Moderna & Argentina \\
\hline $\begin{array}{l}\text { Federico } \\
\text { Sturzenegger }\end{array}$ & Presidente del Banco Ciudad de Buenos Aires & Argentina \\
\hline Flavia Santinoni Vera & $\begin{array}{l}\text { Profesor de derecho y economía, Instituto } \\
\text { Brasiliense de Direito Público }\end{array}$ & Brasil \\
\hline
\end{tabular}




\begin{tabular}{|c|c|c|}
\hline Gabriel Calzada & Presidente del Instituto Juan de Mariana & España \\
\hline Gabriel Zanotti & $\begin{array}{l}\text { Director del Departamento de Economía y } \\
\text { Ciencias Sociales de la ESEADE }\end{array}$ & Argentina \\
\hline Gary Becker (1) & $\begin{array}{l}\text { Premio Nobel en economía en } 1992 . \\
\text { Universidad de Chicago. }\end{array}$ & Estados Unidos \\
\hline $\begin{array}{l}\text { Gerardo } \\
\text { Bongiovanni }\end{array}$ & Presidente de la Fundación Libertad & Argentina \\
\hline Guillermo Yeatts & Presidente de la Fundación Atlas 1853 & Argentina \\
\hline Harald Beyer & $\begin{array}{l}\text { Coordinador académico del Centro de } \\
\text { Estudios Públicos }\end{array}$ & Chile \\
\hline Jaime García Legaz & $\begin{array}{l}\text { Director general del FAES (Fundación para el } \\
\text { Análisis y los Estudios Sociales) }\end{array}$ & España \\
\hline Jorge Avila & $\begin{array}{l}\text { Economista del Centro de Estudios } \\
\text { Macroeconómicos de Argentina }\end{array}$ & Argentina \\
\hline Jorge Edwards & Escritor & Chile \\
\hline Jorge Quiroga & Expresidente de Bolivia & Bolivia \\
\hline Kevin Murphy & $\begin{array}{l}\text { Profesor de economía de la Universidad de } \\
\text { Chicago }\end{array}$ & Estados Unidos \\
\hline Larry Mone & Presidente de The Manhattan Institute & Estados Unidos \\
\hline Leonidas Montes & $\begin{array}{l}\text { Decano de la Escuela de Gobierno de la } \\
\text { Universidad Adolfo Ibáñez }\end{array}$ & Chile \\
\hline $\begin{array}{l}\text { Leonidas } \\
\text { Zelmanovitz }\end{array}$ & Miembro de Liberty Fund & Brasil \\
\hline Lucía Santa Cruz & Decana de la Universidad Adolfo Ibáñez & Chile \\
\hline Luis Larraín & $\begin{array}{l}\text { Director Ejecutivo del Instituto Libertad y } \\
\text { Desarrollo }\end{array}$ & Chile \\
\hline Manuel Hinds & Exministro de economía & El Salvador \\
\hline Marcos Aguinis & Escritor & Argentina \\
\hline Mario Vargas Llosa & Premio Nobel de literatura en 2010 & Perú \\
\hline Martín Krause & $\begin{array}{l}\text { Profesor de Economía de la ESEADE y la } \\
\text { Universidad de Buenos Aires }\end{array}$ & Argentina \\
\hline Mauricio Macri & Intendente de la Ciudad de Buenos Aires. & Argentina \\
\hline Michael Walker & $\begin{array}{l}\text { Presidente de Free Market Research } \\
\text { Foundation }\end{array}$ & Canadá \\
\hline Ramón Parellada & $\begin{array}{l}\text { Tesorero de la Universidad Francisco } \\
\text { Marroquín }\end{array}$ & Guatemala \\
\hline $\begin{array}{l}\text { Ricardo López } \\
\text { Murphy }\end{array}$ & Economista & Argentina \\
\hline $\begin{array}{l}\text { Roberto Cortés } \\
\text { Conde }\end{array}$ & $\begin{array}{l}\text { Historiador, profesor emérito de la } \\
\text { Universidad San Andrés }\end{array}$ & Argentina \\
\hline $\begin{array}{l}\text { Roberto Salinas } \\
\text { León }\end{array}$ & Economista & México \\
\hline Sebastián Edwards & $\begin{array}{l}\text { Economista, profesor de la Universidad de } \\
\text { California }\end{array}$ & Chile \\
\hline Yaron Brook & Presidente de The Ayn Rand Institute & Estados Unidos \\
\hline
\end{tabular}

Fuente: Elaborado en base a la información presente en Mont Pèlerin Society (2011).

(1) No asistió físicamente. Disertó a través de video-teleconferencia desde la Universidad de Chicago. 
En el año 2013, la reunión especial que convocó a los monpelerineses en las Islas Galápagos (Ecuador) llevó la denominación "Evolution, the Human Sciences and Liberty. The Mont Pèlerin Society Special Meeting. Galapagos. 2013". Es de notar, en relación a la propuesta epistemológica hayekiana desarrollada en El orden sensorial (2004) y su vinculación con el desarrollo de las neurociencias analizado en el trabajo de Susana Murillo (2015a), el título, el lugar, el logo (un ave) y la presentación ${ }^{11}$ del evento en tanto elementos que reclaman nuestra atención en relación a la importancia que cobran en la actualidad el desarrollo y propagación de las neurociencias (Mont Pèlerin Society, 2013c).

El crecimiento de la participación de Argentina en la Mont Pèlerin Society que muestra el cuadro 1 no será casual, sino producto de la labor constante de Alberto Benegas Lynch a través del centro que preside.

El Centro de Estudios sobre la Libertad inicia en $1958^{12}$ la publicación periódica de la revista Ideas sobre la Libertad, la cual es acompañada por la edición de numerosos escritos que dicha institución reproduce en español: libros, fracciones de libros aún no terminados de traducir y compilaciones de conferencias o encuentros de monpelerineses de habla hispana. Esta publicación fue reproducida desde

11 Mont Pèlerin Society (2013c), donde se expresa:

What?

This Mont Pelerin Society Special Meeting has the objective to link the concept of evolution to freedom, reinforce the debate that opposes classical liberal society and statism using biology and anthropology as theoretical foundations, and to understand cultural evolution of open societies as a mean to escape from the tribal order. The Universidad San Francisco de Quito (USFQ), from Ecuador, will host this world summit on its Galapagos campus (GAIAS) located on the island of San Cristóbal.

Why?

Friedrich Hayek asserted that: "cultural evolution is not the result of human reason consciously building institutions, but of a process in which culture and reason developed concurrently..." The co-evolution of human preferences and institutions poses serious problems to anyone who promotes policies that supposedly will alter only one of the two. It is the old problem of culture versus institutions. Freedom, property rights, rule of law, how is it that all these elements evolved to promote peace and prosperity? Why some are more prominent only in some societies while in others they are almost inexistent? During this world summit, scholars with training in the natural and social sciences will gather to discuss the evolution of and the current challenges to freedom. Galapagos provides a unique environment for this; it inspired Charles Darwin, more than one hundred fifty years ago, to make his groundbreaking contributions to the biological sciences.

12 Véase Ideas sobre la Libertad (Buenos Aires, Centro de Difusión de la Economía Libre, $\mathrm{N}^{\circ} 1$, diciembre de 1958) 
diciembre de 1958 hasta finales de 1998. En 1984 la ESEADE comienza la edición de la revista Libertas, la cual denomina desde el año 2007 hasta la actualidad Revista de Ideas, Instituciones y Mercados (RIIM) ${ }^{13}$. La ESEADE, de ese modo, da continuidad a las labores iniciadas en el marco del Centro de Difusión de la Economía Libre en materia de difusión escrita de ideas austríacas y montpelerinesas (Gómez, 2012).

El Centro de Estudios Públicos (CEP) acompañó desde Chile la edición de estas revistas argentinas publicadas por la ESEADE y el Centro de Estudios sobre la Libertad. Este instituto fue creado en 1980 sin otra finalidad que el "estudio y difusión de los valores, principios e instituciones que sirven de base a una sociedad libre", publica la revista Estudios Públicos (Centro de Estudios Públicos, 2020, párr. 1).

También compartió camino, a partir de 1984, el Centro de Divulgación del Conocimiento Económico (CEDICE), en Caracas (Centro de Divulgación del Conocimiento Económico, 2020). Institución que define su visión y objetivos del siguiente modo:

Asociación civil, sin fines de lucro fundada en 1984 con el objeto de promover entre los ciudadanos venezolanos las bondades de una sociedad abierta libre, donde se respeten los derechos de propiedad, la libertad económica y los fundamentos de una democracia liberal.

Visión

Nos esforzamos por una Venezuela libre y próspera, donde la vida y la propiedad de sus ciudadanos es protegida.

Misión

Divulgar, formar, investigar y defender los principios del libre mercado y la libertad individual, para construir una sociedad de personas libres y responsables. (Mises von, 2011, p. 4).

El Centro de Difusión de la Economía Libre, por su parte, se reconoce ortodoxo y entiende que la ortodoxia ha sido trazada por la tradición austríaca en su línea más dura o propia, integrada por Carl Menger,

13 Puede consultarse el contenido de dicha publicación en la página de la Biblioteca de la ESEADE: www.eseade.edu.ar/riim/libertas/. En esa página web se encuentran online todos los ejemplares de Libertas y RIIM. 
Eugen Bömh Bawerk y Ludwig von Mises. Así lo afirma Ideas sobre la Libertad (1960) en su nota editorial:

Una HONDA Y ARRAIGADA CONVICCIÓN nos coloca así en la línea de pensamientos ortodoxa, trazada en este materia por los grandes maestros, fundadores y continuadores de la llamada moderna escuela vienesa, con Böhm- Bawerk, Menger y von Mises a la cabeza. (p. 2).

Por tanto, los autores y las obras generales que son difundidas por el CDEL pueden considerarse inspiradas, propias o en línea con la escuela austríaca de economía y sus principios. Asimismo, no todas sus publicaciones tienen una filiación específica a dicha escuela. Por ello, entendemos que el estudio de dicho centro y sus publicaciones pueden entenderse como el primer arribo del pensamiento austríaco y montpelerinés a la Argentina, aunque ello no nos impide visualizar distintas vertientes presentes en su devenir.

\section{Un dato para la actualidad}

En diciembre de 2015, la Fundación Libertad -par encargada de organizar en el año 2011 el encuentro regional de la Sociedad denominado "Regional Meeting. Buenos Aires: the populist challenge to Latin American liberty" - otorgó el premio Libertad al periodista Jorge Lanata, tras la derrota electoral del Frente para la Victoria en las elecciones presidenciales. Este espacio político partidario, liderado por la presidenta saliente en aquel momento, Cristina Fernáadez de Kirchner,-fue intensamente criticado por las instituciones y actores vinculados a la Mont Pèlerin Society, a causa de sus tendencias "populistas" y el periodista antes mencionado abocó su programa "Periodismo para Todos" a denunciar y caricaturizar a los integrantes de la citada agrupación política (Lanata, 2015; Fundación Libertad, 2016).

Entre los oradores del evento, además del congratulado periodista, se destacan los saludos del empresario Mauricio Macri, quien triunfase en las elecciones presidenciales antes referidas y de Mario Vargas Llosa, otro reconocido integrante de la Sociedad (Fundación Libertad, 2015a, 2015b).

Cabe destacar la figura de Jorge Lanata en Argentina como uno de los periodistas más reconocidos por su férrea oposición al gobierno 
"populista" saliente así como por la efectividad de su programa televisivo en la consecución de la divulgación social de denuncias del gobierno anterior y del chavista en Venezuela. El propio Lanata ha señalado en entrevistas que uno de los reconocimientos que le ha formulado el público de su programa es su aporte en la derrota del gobierno kirchnerista. Agregamos a ello que la carrera de este periodista encontró como destino posterior las tierras de Miami, donde se le encargó la edición de programas del estilo de su conocido "Periodismo para Todos", pero ahora con objeto de extender su cobertura de críticas al resto de América Latina (Jmortizz77, 2016).

López Murphy dicta en los primeros meses del año 2016, en consonancia con lo anterior, conferencias en distintos lugares y universidades latinoamericanas bajo la denominación "¿Cómo derrotar al populismo?" mediante la organización de RELIAL, red que nuclea actualmente distintos centros latinoamericanos pares de Fundación Libertad (Hey Event, 2016).

\section{Discusión y conclusiones}

Lo relatado anteriormente nos ha permitido observar uno de los primeros arribos del neoliberalismo en el espacio local argentino y su vinculación con otros centros pares en Latinoamérica. Un ingreso caracterizado por la prominencia de la mirada o participación de exponentes de la escuela austríaca de economía frente a la distintiva participación de la escuela de Chicago que caracteriza al fenómeno a partir los años 70. Cuestión que, vimos, obedece a los cambios acaecidos en la composición de la Mont Pèlerin Society en el devenir del siglo XX y que son atendidos en investigaciones previas y en la propia historia oficial de la Sociedad.

El arribo puede remitirse a la llegada al poder de la revolución libertadora, una gestión presidencial que se caracterizó por su carácter no democrático y autoritario, por su proscripción al peronismo, por su enfrentamiento a las fracciones obreras, por la inauguración de su ascenso en el bombardeo de plaza de Mayo y por la articulación sus altos mandos a miembros dirigentes de la Mont Pèlerin Society. Ejemplo de ello fueron las vinculaciones establecidas ente Alberto Benegas Lynch y Eugenio Aramburu con Friedrich von Hayek. 
La influencia de la Mont Pèlerin Society en tierras argentinas no se reduce al funcionariado o cuerpo político del estado, sino que se expande a distintas instituciones que detentan un tipo de enunciación particular, el saber académico, y que, mediante el financiamiento de sectores empresarios, destinan sus esfuerzos a promover la difusión y expansión de un determinado cuerpo discursivo. Aquellas son, fundamentalmente, instituciones que actualmente estilan denominarse tanques de pensamientos. Ese es el caso del Centro de Difusión de la Economía Libre (CDEL), la Escuela Superior de Economía y Administración de Empresas (ESEADE), la Fundación Libertad y la Red Liberal de América Latina (RELIAL).

Estas instituciones, en primer lugar, se han vinculado en el pasado y se vinculan en la actualidad con distintos actores políticos, entre los que podemos destacar las figuras de Eugenio Aramburu, Federico Pinedo, Mauricio Macri y el mismo Alberto Benegas Lynch, funcionario que en vigencia de la revolución libertadora se desempeñó como embajador argentino en los EEUU; en segundo lugar, cuentan con la labor y colaboración de periodistas como Jorge Lanata y de medios de comunicación como los periódicos La Prensa y La Nación; en tercer lugar, se financian con el aporte de grandes empresarios como Amalia Sara Lacroze de Fortabat y de instituciones empresariales como la Bolsa de Comercio de Buenos Aires; en cuarto lugar, intentan vincularse a ámbitos universitarios como la Facultad de Ciencias Económicas de la Universidad de Buenos Aires (UBA) y la Universidad Autónoma de México (UNAM), en tanto, estos últimos, máximos representante del saber científico.

Para finalizar, este trabajo ha presentado algunos elementos que permiten en alguna medida vislumbrar el modo en que la Mont Pèlerin Society ingresa en Argentina. Arribo en el cual asume estrategias y objetivos similares a los que se propone en el espacio internacional. Esto es, vincular ámbitos empresariales, en primera instancia, con instituciones y actores que se desempeñan como productores y difusores discursivos y, en segunda instancia, con gestores de política públicas. Tareas que desempeña con objeto de influir en el devenir de las naciones, siendo más precisos, con el objeto de facilitar la primacía de un modo de ordenamiento socíal que la Sociedad ha dado en llamar neoliberalismo. 


\section{Referencias}

Anderson, P. (2003). Más allá del neoliberalismo: lecciones para la izquierda. En E. Sader \& P. Gentilli (Eds.), La trama del neoliberalismo. Mercado, crisis y exclusión social (pp. 192-194). Buenos Aires: Consejo Latinoamericano de Ciencias Sociales ,CLACSO. Recuperado de http://bibliotecavirtual.clacso. org.ar/ar/libros/trama/ander.rtf.

Astarita, R. (2010). Economía política de la dependencia y el subdesarrollo: tipo de cambio y renta agraria en la Argentina. Bernal, Argentina: Universidad Nacional de Quilmes.

Basualdo, E. (2006). Estudio de historia económica argentina desde mediados de siglo XX a la actualidad. Buenos Aires: Facultad Latinoamericana de Ciencias Sociales (FLACSO), Siglo XXI.

Basualdo, E., Santarcángelo, J., Wainer, A., Russo, C. \& Perrone, G. (2016). El Banco de la Nación Argentina y la dictadura. El impacto de las transformaciones económicas y financieras en la política crediticia (1976-1983). Buenos Aires: Siglo XXI.

Belenky, S. (1984). Frondizi y su tiempo. Buenos Aires: Centro Editor de América Latina.

Beltrán, G. (2005a). Formación profesional y producción intelectual en tiempos de cambio político. Las carreras de sociología y economía de la Universidad de Buenos Aires durante los años noventa. Buenos Aires: Consejo Latinoamericano de Ciencias Sociales (CLACSO).

Beltrán, G. (2005b). Los intelectuales liberales: poder tradicional y poder pragmático en la argentina reciente. Buenos Aires: Eudeba.

Benegas Lynch, A. (1978). Discursos inaugurando el ciclo de conferencias del Dr. Friedrich A. Hayek. En F. Hayek (Ed.), Temas de la hora actual (pp. 7-12). Buenos Aires: Bolsa de Comercio de Buenos Aires.

Benegas Lynch, A., García Belsunce, H., Loncán, E. \& Luzzetti, C. (1979). Conferencias sobre el pensamiento de Ludwig von Mises. Buenos Aires: Fundación Bolsa de Comercio de Buenos Aires.

Benegas Lynch, A. (2007). Bases de la Constitución de ESEADE. Recuperado de https://www.eseade.edu.ar/institucional-2/ carta-del-rector/bases-de-la-constitucion-de-eseade/

Büren de, M. P. (2013a). Mont Pèlerin Society en la articulación del discurso neoliberal. En H. Ramirez (Coord.), O neoliberalismo 
sul-americano em clave transnacional: enraizamento, apogeu e crise (pp. 118-143). São Leopoldo: Oikos, Edunisinos.

Büren de, M. P. (2013b). La disciplina económica en la construcción del sentido común, un acercamiento desde el instrumental analítico gramsciano. Astrolabio. Nuevo Tiempo, (10), 45-81. Recuperado de:https://revistas.psi.unc.edu.ar/index.php/ astrolabio/article/view/3392/4497

Büren de, M. P. (2014). La escuela austríaca de economía, expansión y difusión de sus ideas fuerza. Argentina 1959-1989 (Tesis doctoral inédita). Universidad de Buenos Aires, Buenos Aires.

Büren de, M. P. (2015a). Mont Pèlerin Society, un espacio de articulación. En S. Murillo (Coord), Neoliberalismo y gobiernos de la vida. Diagrama global y despliegues en Argentina y América Latina (pp. 77-90). Buenos Aires: Biblos.

Büren de, M. P. (2015b). Neoliberalismo, mecanismos de difusión de su construcción discursiva, una lectura desde el herramental analítico foucaultiano. Anuario de investigaciones del Centro Cultural de la Cooperación Floreal Gorini, (5), 1-33. Recuperado de https://ri.conicet.gov.ar/handle/11336/110665

Büren de, M. P (2017). Antinomia economía positiva-economía normativa, apuntes para el análisis de la conformación de un régimen de veridicción. En M. Campana y J. Giavedoni (Comps.), Debates sobre Estado, gobierno y control social: revitalizar las prácticas de resistencia (pp. 169-186). Rosario: Pegues.

Büren de, M. P. (2018). Ludwig von Mises, disputas de significación en la estrategia neoliberal. Revista de la Carrera de Sociología, (8), 450-494. Recuperado de https://publicaciones. sociales.uba.ar/index.php/entramadosyperspectivas/issue/ view/330

Büren de, M. P. (2019). Neoliberalismo, el secreto como estrategia. RevIISE. Revista de Ciencias Sociales y Humanas, 13, 77-90. Recuperado de http://www.ojs.unsj.edu.ar/index.php/reviise/ issue/view/20/showToc

Büren de, M. P (2020). Contra ofensiva neoliberal. La escuela austriaca de economía en el centro estratégico de la disputa. Buenos Aires: Consejo Latinoamericano de Ciencias Sociales (CLACSO), Instituto de Investigaciones Gino Germani. Recuperado de http://www.clacso.org.ar/libreria-latinoamericana/contador/sumar_pdf.php?id_libro=2223 
Castellani, A. (2006). Estado, empresas y empresarios. La relación entre intervención económica estatal, difusión de ámbitos privilegiados de acumulación y desempeño de las grandes firmas privadas. Argentina 1966-1988 (Tesis doctoral inédita). Universidad de Buenos Aires, Buenos Aires.

Centro de Divulgación del Conocimiento Económico. (2020). About us. Recuperado de https://cedice.org.ve/about-us-2/

Centro de Estudios Públicos. (2020).¿Qué es el CEP?. Recuperado de https://www.cepchile.cl/cep/que-es-el-cep

Denord, F. (2002). Le prophète, le pèlerin et le missionnaire. La circulation internationale du néo-libéralisme et ses acteurs. Actes de la Recherche en Sciences Sociales, (145), 9-20.

Fernández López, M. (2001). El pensamiento económico (1914-1983). En Academia Nacional de la Historia (Ed.), Nueva historia de la nación argentina (Vol. 8, pp. 499-523). Buenos Aires: Planeta.

Fernández López, M. (2008). Economía y economistas argentinos 16002000. Buenos Aires: Consejo Profesional de Ciencias Económicas de la Ciudad de Buenos Aires.

Foucault, M. (2007a). El nacimiento de la biopolítica. Curso del Collège de France (1978-1979). Buenos Aires: Fondo de Cultura Económica.

Foucault, M. (2007b). La arqueología del saber. Buenos Aires: Siglo XXI.

Fundación Libertad. (9 de diciembre de 2015a). Saludos a Jorge Lanata en la entrega del Premio Libertad [Video]. Recuperado de https://www.youtube.com/watch?time_ continue $=2 \& v=$ ssChyKfJyHE

Fundación Libertad. (9 de diciembre de 2015b). Saludo del Presidente Mauricio Macri a Fundación Libertad en su cena anual [Video]. Recuperado de https://www.youtube.com/ watch?v=whmTJUsu9Wc

Fundación Libertad. (5 de marzo de 2016). Balance 2015. Rosario: Red libertad. Fundación internacional para la libertad. Recuperado de https://issuu.com/mandyva/docs/fl_balance_2015_final

Gómez, A. (2012). ESEADE 1978-2008. Recuperado de http://www.eseade.edu.ar/institucional/institucional/eseade-1978-2008.html

Gramsci, A. (2008). Los intelectuales y la organización de la cultura. Buenos Aires: Nueva Visión. 
Grondona, A. (2011). Las voces del desierto. Aportes para una genealogía del neoliberalismo como racionalidad de gobierno en la Argentina (1955-1975). El búho y la alondra, (13). Recuperado de https://www.centrocultural.coop/revista/13/lasvoces-del-desierto-aportes-para-una-genealogia-del-neoliberalismo-como-racionalidad

Grondona, A. (2012). “Tradición" y "traducción”: un estudio de las formas contemporáneas del gobierno de las poblaciones desempleadas en la Argentina (Tesis doctoral). Universidad de Buenos Aires, Argentina. Recuperado de https://www.centrocultural.coop/publicaciones/tradicion-y-traduccion-unestudio-de-las-formas-contemporaneas-del-gobierno-de-las

Hartwell, R. (1995). A history of the Mont Pèlerin Society. Indianapolis: Liberty Fund.

Harvey, D. (2007) La breve historia del neoliberalismo. Madrid: Akal.

Hayek von, F. (1961). Los sindicatos y la ocupación obrera. Buenos Aires: Centro de Estudios sobre la Libertad.

Hayek von, F. (1978). Temas de la hora actual. Buenos Aires: Bolsa de Comercio de Buenos Aires.

Hayek von, F. (2004). El orden sensorial. Los fundamentos de una psicología teórica. Madrid: Unión Editorial.

Hayek von, F. (2008). Los fundamentos de la libertad. Madrid: Unión.

Heredia, M. (2004). El proceso como bisagra. Emergencia y consolidación del liberalismo tecnocrático: FIEL, FM y CEMA. En A. Pucciarelli (Comp.), Empresarios, tecnócratas y militares. La trama corporativa de la última dictadura (pp. 312-382). Buenos Aires: Siglo XXI.

Heredia, M. (2006). La demarcación de la frontera entre economía y política en democracia. Actores y controversias en torno a la política económica de Alfonsín. En A. Pucciarelli (Comp.), Los años de Alfonsín. ¿El poder de la democracia o la democracia del poder? (pp. 153-198). Buenos Aires: Siglo XXI.

Hey Event. (2016). Argentina ¿Cómo vencer al populismo? Recuperado de http://heyevent.com/event/gl3elqh6q3k46a/argentinacomo-vencer-al-populismo

Ideas sobre la Libertad. (1958). Editorial. Ideas sobre la Libertad (1), 1-63. Ideas sobre la Libertad. (1960). Por qué somos ortodoxos. Ideas sobre la Libertad, (5), 2-3. 
Jmortizz77. (20 de marzo de 2016). Jorge Lanata en La Noche de Mirtha (Programa del 19 de marzo de 2020) [Video]. Recuperado de https://www.youtube.com/watch?v=TAwa5hg_wMQ

La Nación. (20 de febrero de 1999). Alberto Benegas Lynch. El sepelio. La Nación. Recuperado de http://www.lanacion.com. ar/128581-alberto-benegas-lynch

La Nación. (23 de Noviembre de 2007). Federico Zorraquín. El fallecimiento. La Nación. Recuperado de https://www.lanacion. com.ar/economia/federico-zorraquin-nid964815/

Lanata, J. (2015). Discurso de Jorge Lanata. Premio Libertad 2015. Recuperado de https://grupojovenfl.files.wordpress. com/2015/12/discurso-lanata-premio-libertad-2015.pdf

Liberaal Archief (2004). Mont Pèlerin Society (1947- ). Inventory of the General Meeting Files (1947-1998). Recuperado de: http:// rybn.org/thegreatoffshore/THE\%20GREAT\%20OFFSHORE/1.ENCYCLOPEDIA/MONT\%20PELERIN\%20SOCIETY,\%201947/DOCS/Inventory\%20of\%20the\%20General\%20 Meeting\%20Files\%20(1947-1998).pdf

Mises von, L. (1959). Seis conferencias en Buenos Aires. Crónicas publicadas por el diario "La Prensa" de Buenos Aires. Buenos Aires: Centro de Difusión de la Economía Libre.

Mises von, L. (1978). Tres mensajes. Buenos Aires: Eundación Bolsa de Comercio de Buenos Aires.

Mises von, L. (1979). La mentalidad anticapitalista. Buenos Aires: Bolsa de Comercio de Buenos Aires.

Mises von, L. (1996). Sobre liberalismo y capitalismo. (Vol. 2). Barcelona: Folio, Unión Editorial.

Mises von, L. (2011). Ideas sobre la libertad, la sabiduría de Ludwig von Mises. [Páginado de: http://libreriacedice.org.ve/wpcontent/uploads/2019/06/La-Sabiduria-de-Ludwig-vonMises.pdf

Mises von, M. (1996). Prólogo de Seis Lecciones sobre el Capitalismo. En L. von Mises (Ed.), Sobre liberalismo y capitalismo (Vol. 2, (p. 277-278). Barcelona: Folio, Unión Editorial.

Mont Pèlerin Society. (2011). Programa. En Mont Pèlerin Society Regional Meeting 2011 Buenos Aires. Fundación Libertad. [Página institucional]. Recuperado de http://www.mpsargentina.org/programcomittee.html. 
Mont Pèlerin Society. (2013a). The Mont Pèlerin Society. Recuperado de https://www.montpelerin.org/montpelerin/home.html

Mont Pèlerin Society. (2013b). Past Meetings. Recuperado de https:// www.montpelerin.org/montpelerin/meetings.html

Mont Pèlerin Society. (2013c). Mont Pèlerin Society Special meeting: evolution, the human sciences and liberty. Galápagos. Recuperado de http://www.usfq.edu.ec/eventos/mps_galapagos/Paginas/default.aspx

Morresi, S. (2008). La nueva derecha argentina: la democracia sin política. Los Polvorines, Argentina: Universidad Nacional General Sarmiento, Biblioteca Nacional.

Murillo, S. (2015a). Biopolítica y procesos de subjetivación en la era neoliberal. En S. Murillo (Comp.), Neoliberalismo y gobiernos de la vida. Diagrama global y sus configuraciones en Argentina y América latina (pp. 17-40). Buenos Aires: Biblos.

Murillo, S. (Coord.). (2015b). Neoliberalismo y gobiernos de la vida. Diagrama global y despliegues en Argentina y América Latina. Buenos Aires: Biblos.

Neiburg, F. \& Plotkin, M. (Comp.) (2004). Intelectuales y expertos: la constitución del conocimiento social en Argentina. Buenos Aires: Paidós.

Novaro, M. (2010). Historia de la Argentina 1955-2010. Buenos Aires: Siglo XXI.

Pucciarelli, A. (Coord.) (2004). Empresarios, tecnócratas y militares. La trama corporativa de la última dictadura. Buenos Aires: Siglo XXI.

Reig Albiol, J. \& Sennholz, H. (1977). La teoría de la explotación. Buenos Aires: Fundación Bolsa de Comercio de Buenos Aires.

Roig, A. (2007). La monnaie impossible: la convertibilité argentine de 1991 (Tesis doctoral inédita). L'École des Hautes Etudes en Sciences Sociales, Paris.

Rothbard, M. (1979). Moneda, libre y controlada. Buenos Aires: Fundación Bolsa de Comercio de Buenos Aires.

Rouquié, A. (1982). Poder militar y sociedad civil en la Argentina II (1943-197). Buenos Aires: Emecé.

Rozenwurcel, G. Bezchinsky, G. \& Chatruc Rodríguez, M. (2007). La enseñanza de economía en Argentina. Buenos Aires: Universidad Nacional de San Martín. 
Rubinich, L. (2001). La conformación de un clima cultural. Neoliberalismo y universidad. Buenos Aires: Universidad de Buenos Aires; Libros del Rojas.

Schorr, M. (2006). Cambios en la estructura y el funcionamiento de la industria argentina entre 1976 y 2004. Un análisis sociohistórico y de economía política de la evolución de las distintas clases sociales y fracciones de clase durante un periodo de profundos cambios estructurales (Tesis doctoral inédita). Universidad de Buenos Aires, Buenos Aires.

Sennholz, H. (1977). Problemas económicos de actualidad. Buenos Aires: Centro de Estudios sobre la Libertad, Bolsa de Comercio de Buenos Aires.

Sztulwark, S. (2003). El estructuralismo latinoamericano. Fundamentos y transformaciones del pensamiento económico de la periferia. Buenos Aires: Universidad Nacional de General Sarmiento.

Torrado, S. (1994). Estructura social de la Argentina, 1945-1983. Buenos Aires: Ediciones de la Flor.

Torrado, S. (2006). Familia y diferenciación social. Cuestiones de método. Buenos Aires: Editorial Universitaria de Buenos Aires.

Unzué, M. (2020). Profesores, científicos e intelectuales: la Universidad de Buenos Aires de 1955 a su Bicentenario. Buenos Aires: Consejo Latinoamericano de Ciencias Sociales (CLACSO), Instituto de Investigaciones Gino Germani. Recuperado de http://biblioteca.clacso.edu.ar/Argentina/iigguba/20200508051556/Universidad-Unzue.pdf

Velasco, G.; Reig, J. \& Benegas Lynch, A. (1961). Deliberaciones sobre la Libertad. Buenos Aires: Instituto Venezolano de Análisis Económico y Social, Centro Venezolano de Estudios Sobre la Libertad.

Vuolo lo, R. (2000). Alternativas: la economía como cuestión social. Buenos Aires: Altamira. 